\title{
Characterization of Catalyst-nanomaterials with Myriad Modalities of Transmission Electron Microscopy
}

Dalaver H. Anjum ${ }^{1 *}$, Walid AlMaksoud ${ }^{2}$, Rohit K. Rai ${ }^{2}$, Bedour E. Alsabban ${ }^{3}$, Natalia Morlanes ${ }^{2}$ and Jean -M. Basset ${ }^{2}$

${ }^{1 .}$ King Abdullah University of Science \& Technology (KAUST), Core Labs, Thuwal, Saudi Arabia.

2. KAUST Catalysis Center, King Abdullah University of Science \& Technology (KAUST), Thuwal, Saudi Arabia.

3. SABIC Corporation Research and Innovation Center, Thuwal, Saudi Arabia.

* Corresponding author: dalaver.anjum@kaust.edu.sa

Catalysts are important to many chemistry based reactions including the reduction of $\mathrm{CO}_{2}$, the production of $\mathrm{H}_{2}$, and activation of $\mathrm{N}_{2}$ [1]. In all cases, the selection of catalyst-materials should be made after a complete understanding of their structure-property relationships. The main reason for doing this is because the catalytic activity of materials strongly depends on their properties e.g. their morphology, internal structure, elemental distribution, and chemical states of elements. Furthermore, catalyst-materials are rapidly being replaced these days with so-called nano-catalysts or nanomaterials whose structural properties are often very different than their bulk-counterparts. That is why, it becomes even more essential to re-examine the structure-property relationships between the material properties and chemical/catalytic activity. Moreover it must be carried out with an elaborate technique, such as transmission electron microscopy (TEM), capable of providing the needed-information at the underlying nanoscale dimensions [2]. The objective of the work presented herein is therefore to perform a holistic TEM-analysis of a nano-catalyst material. For this purpose, a specific nano-catalyst which is designed for the activation of $\mathrm{N}_{2}$ gas, is considered herein. It is basically a nanocomposite of cesium (Cs) loaded onto iron-carbon $(\mathrm{Fe}-\mathrm{C})$ material. The synthesis of this nanomaterial was carried out in two processes namely the pyrolysis and impregnation. TEM-analysis of the nano-catalyst was then done with a probe-corrected electron microscope of model Titan 80-300 ST that was also equipped with a Quantum-GIF of model 966. Furthermore, the analysis was performed by operating the microscope at the accelerating voltage of 300 $\mathrm{kV}$. Throughout the analysis, both electron beam current and exposure time were adjusted to minimize the beam-induced damage to the specimens.

Figure 1 A contains an image of above-mentioned nano-catalyst which was acquired in bright-field TEM (BF-TEM) mode. The selected area electron diffraction (SAED) and X-ray energy dispersive (EDS) from the corresponding region are also shown therein as inserts. This analysis revealed that the catalyst was indeed a nanocomposite containing all elements in the expected composition and furthermore at least one of its components was crystalline. Three-dimensional (3d) imaging was important for knowing the fraction of nonmaterial participating to catalytic reaction and hence was accomplished with employing electron tomography (ET) analysis of these specimens (Figure 1B). It can be seen from therein that the typical thickness of the catalyst nanomaterial was in the range couple of hundred nanometers. In other words, these $3 \mathrm{~d}$ results imply that almost whole nanomaterial (both surface and bulk) would be catalytically active in the reaction. Dark-field scanning TEM (DF-STEM) analysis, shown in Figure 2 A and B, basically confirms the same nanocomposite structure on synthesized material. Additionally, it reveals a core-shell structure of nanoparticles and hence creating a demand of performing the elemental mapping analysis. It 
has been thus done with spectrum-imaging (SI) analysis of samples in which electron energy-loss spectroscopy (EELS) analysis were done simultaneously with the acquisition of DF-STEM images. The acquired SI datasets were then post-processed in Gatan Microscopy Suite (GMS v3.1) and it allowed to generate the elemental maps (shown as red-green-blue (RGB) composites in Figure $2 \mathrm{C}$ and D). Both RGB maps reveal the formation oxidized Fe nanoparticles (Fe-NPs) which were lying in Cs and C matrix. Furthermore, the Cs-metal was forming a shell around core-shell oxidized Fe-NPs. The determination of chemical or valence states of Fe-NPs, shown as insert in Figure 2D, was done by analyzing the ratio of Fe-L2 and Fe-L3 peaks of Fe-L23 edge. It showed that the surface of Fe-NPs was in + 2 valence state while the core was in reduced metal state. In conclusion, modern TEM instruments enable in carrying out a holistic nano-scale characterization of materials used for catalysis applications.

\section{References:}

[1] VC Jacques, Catalysts 7 (11), 341 (2017) p. 1.

[2] S Dong, Green Energy \& Environment 2 (2017), p.70.

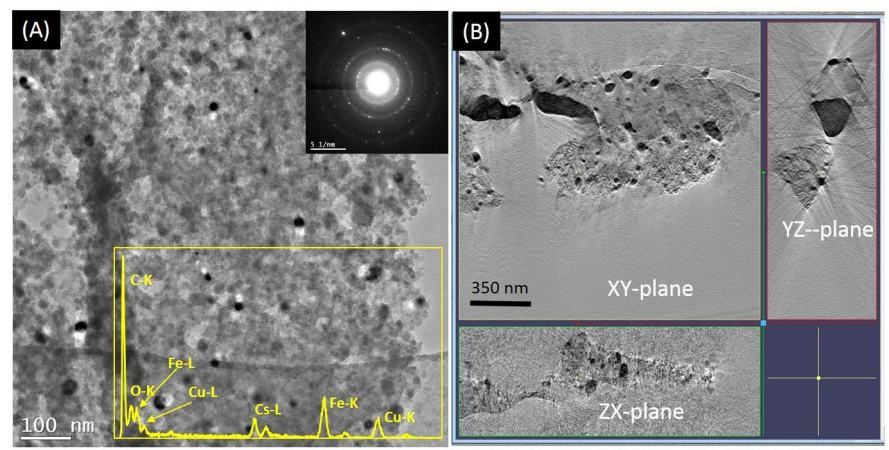

Figure 1. TEM-analysis of a catalyst nanomaterial
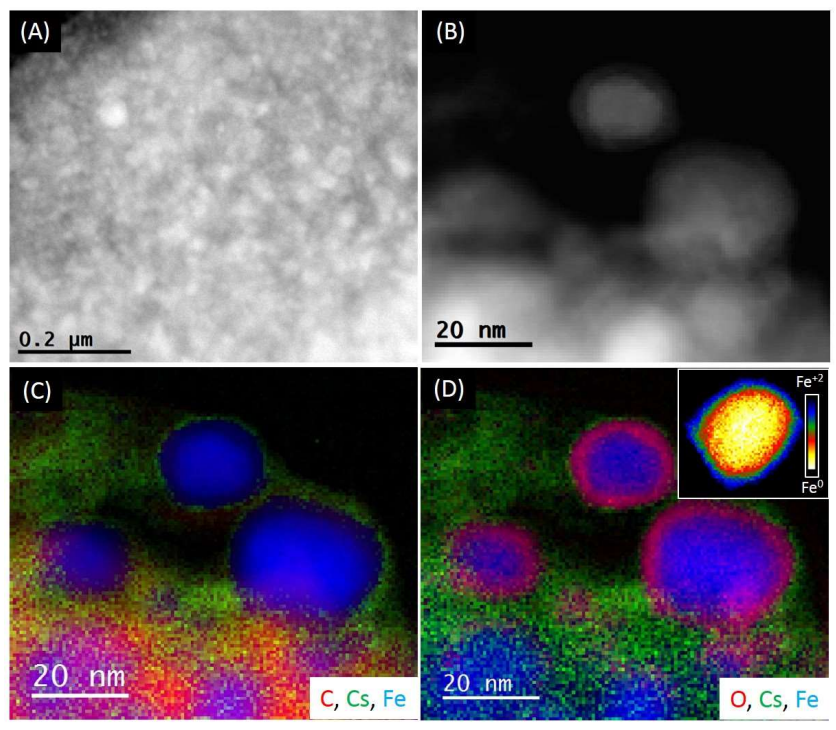

Figure 2. Elemental and chemical analyses of a catalyst nanomaterial 